\title{
Statistically Robust Cooperative Beamforming for Cognitive Radio Networks
}

\author{
Sudhir Singh*, Paul D. Teal ${ }^{\dagger}$, Pawel A. Dmochowski ${ }^{\dagger}$ and Alan J. Coulson* \\ * Callaghan Innovation, Lower Hutt, New Zealand \\ $\dagger$ School of Engineering and Computer Science, Victoria University of Wellington, Wellington, New Zealand \\ Email:\{sudhir.singh,alan.coulson\}@ callaghaninnovation.govt.nz, \{paul.teal,pawel.dmochowski\}@vuw.ac.nz
}

\begin{abstract}
We consider a cognitive radio (CR) relay network consisting of a cognitive source, a cognitive destination and a number of cognitive relay nodes that share spectrum with a primary transmitter and receiver. Due to poor channel conditions, the cognitive source is unable to communicate directly with the cognitive destination and hence employs the cognitive relays for assistance. We assume that the $C R$ has a very loose cooperation with the primary network and therefore, only partial channel state information is available. Under these assumptions, we propose a new statistically robust $\mathrm{CR}$ cooperative relay beamformer where either the total relay transmit power or the cognitive destination signal-to-interference-and-noise ratio (SINR) is optimised subject to primary receiver outage probability constraint. We formulate the robust total relay power and the cognitive destination SINR optimisation problems as a convex second order cone program and a convex feasibility problem, respectively, that provide near optimum results. We also present efficient iterative algorithms that provide the optimum results.
\end{abstract}

\section{INTRODUCTION}

A number of papers have appeared on various aspects of cognitive radio (CR) systems, including fundamental information theoretic capacity limits (see, eg., $[1,2])$. In an underlay $\mathrm{CR}$ system the secondary users (SUs) protect the primary user (PU) by regulating their transmissions to maintain the PU receiver interference below a defined threshold level. The limits on this received interference level at the PU receiver can be imposed by an average/peak constraint, or a minimum signal-to-interference-and-noise ratio (SINR). The advantage of using an SINR-based PU protection mechanism as opposed to $\mathrm{PU}$ interference temperature based protection, is that it removes the constant interference threshold, thus benefiting the SUs when the PU link is strong.

Cooperative relaying has been shown to significantly improve performance in wireless systems [3-6]. Relay nodes are cooperatively able to form a virtual antenna array to exploit diversity and provide increased gains in capacity through distributed beamforming. The concept of user cooperation as a new form of diversity for mobile communication systems was introduced in [3]. Using a convex optimisation framework, the distributed beamforming problem for relay networks has been analysed in $[4,5]$. A relay system equipped with multiple antennas was studied in [6].

Due to its advantages, cooperative beamforming has attracted much interest in CR research [7-9]. A typical spectrum sharing cognitive relay network consists of a cognitive source, a cognitive destination and a number of cognitive relay nodes that co-exist with a primary transmitter (PU-Tx) and receiver (PU-Rx). One of the challenges of spectrum sharing is guaranteeing quality of service (QoS) to the PU. Generally the goals of the CR are not compatible with the goals of the PU, for instance, increasing SU power to increase SU capacity will tend to increase interference to the PU. Beamforming (conventional or cooperative) is seen as a way to alleviate some of these issues, since the $\mathrm{SU}$ or CR relays can direct its power away from the PU receiver. Design of CR cooperative relay beamformers under the assumption of full channel state information (CSI) has been the subject of investigation in [79]. Unfortunately, in practice full CSI is seldom available. To this extent, several works have appeared on the design of robust cooperative relay beamformers based on worst case performance optimisation [5, 10, 11]. Unfortunately, solutions obtained through worst case approach can be overly conservative because the true probability of worst case errors may be extremely low [12].

In this paper, we consider a $\mathrm{CR}$ relay network with a very loose cooperation with the primary network. We assume that only mean channel powers of the PU transmitter to PU receiver and the relays to $\mathrm{PU}$ receiver links are available. We propose a new statistically robust $\mathrm{CR}$ cooperative relay beamformer where either the total relay transmit power or the cognitive destination SINR is optimised subject to PU receiver outage probability constraint. We formulate the robust total relay power and the cognitive destination SINR optimisation problems as a convex second order cone program (SOCP) [13] and a convex feasibility problem, respectively, that provide near optimum results. We also present efficient iterative algorithms that provide the optimum results. Relay transmit power and PU receiver and cognitive destination SINR cumulative distribution functions (CDFs) are obtained through solutions of our optimisation problems.

Notation: Upper (lower) bold face letters are used for matrices (vectors); $(\cdot)^{*},(\cdot)^{T},(\cdot)^{H}, \mathbb{E}\{\cdot\}$ and $\|\cdot\|$ denote complex conjugate, transpose, Hermitian transpose, expectation and Euclidean norm, respectively. $|\cdot|^{2}$ denotes the magnitude squared operator for scalars and element-wise magnitude squared for vectors. $\operatorname{tr}(\cdot), \mathcal{C}^{R \times 1}, \mathcal{C}^{R \times R}, \odot, \Re\{\cdot\}$ and $\Im\{\cdot\}$ denote the matrix trace operator, space of $R \times 1$ vectors with complex entries, space of $R \times R$ matrices with complex entries, elementwise product between vectors, the real part and the imaginary part. $\mathbf{W} \succeq 0$ denotes that $\mathbf{W}$ is a positive 


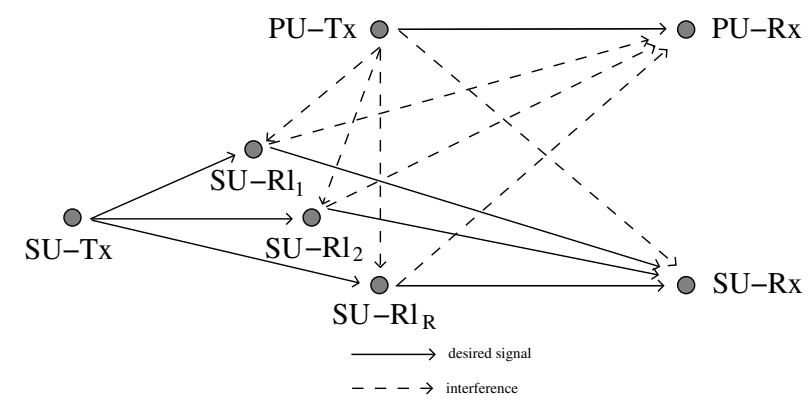

Fig. 1. System Model

semidefinite matrix. The notation $\mathbf{x} \sim \mathcal{N}_{\mathcal{C}}(\mathbf{m}, \boldsymbol{\Sigma})$ states that $\mathrm{x}$ contains entries of complex Gaussian random variables, with mean $\mathbf{m}$ and covariance $\boldsymbol{\Sigma}$.

\section{SySTEM MOdEL}

As shown in Fig. 1, we consider a cognitive radio relay network which consists of a secondary transmitter (SU-Tx), a secondary receiver (SU-Rx), $R$ secondary relay (SU-Rl) nodes and a pair of primary transmitter and receiver. The SU-Tx communicates with the SU-Rx only via SU-Rls. Since the $\mathrm{PU}$ and SU systems use the same frequency band, the PU$\mathrm{Rx}$ experiences interference from the SU-Rl and both SURl and SU-Rx experience interference from the PU-Tx. Each transmitter and receiver in the system are assumed to be equipped with a single antenna.

Independent, point-to-point, flat Rayleigh fading channels are assumed for all links in the network. Let $h_{\mathrm{pp}}, h_{\mathrm{pr}}^{(i)}, h_{\mathrm{ps}}$, $h_{\mathrm{sr}}^{(i)}, h_{\mathrm{rs}}^{(i)}$ and $h_{\mathrm{rp}}^{(i)}$ denote the channel coefficients of the PUTx to PU-Rx, PU-Tx to SU-Rl $i$, PU-Tx to SU-Rx, SUTx to SU-Rl $i$, SU-Rl $i$ to SU-Rx and SU-Rl $i$ to PURx links, respectively. The instantaneous channel powers of these links are represented by $g_{\mathrm{pp}}=\left|h_{\mathrm{pp}}\right|^{2}, g_{\mathrm{pr}}^{(i)}=\left|h_{\mathrm{pr}}^{(i)}\right|^{2}$, $g_{\mathrm{ps}}=\left|h_{\mathrm{ps}}\right|^{2}, g_{\mathrm{sr}}^{(i)}=\left|h_{\mathrm{sr}}^{(i)}\right|^{2}, g_{\mathrm{rs}}^{(i)}=\left|h_{\mathrm{rs}}^{(i)}\right|^{2}$ and $g_{\mathrm{rp}}^{(i)}=\left|h_{\mathrm{rp}}^{(i)}\right|^{2}$. Furthermore, we assume that the channel powers for all links are independent and identically distributed (i.i.d.) and are governed by their corresponding parameters $\mathbb{E}\left\{g_{\mathrm{pp}}\right\}=\Omega_{\mathrm{pp}}$, $\mathbb{E}\left\{g_{\mathrm{pr}}^{(i)}\right\}=\Omega_{\mathrm{pr}}^{(i)}, \mathbb{E}\left\{g_{\mathrm{ps}}\right\}=\Omega_{\mathrm{ps}}, \mathbb{E}\left\{g_{\mathrm{sr}}^{(i)}\right\}=\Omega_{\mathrm{sr}}^{(i)}, \mathbb{E}\left\{g_{\mathrm{rs}}^{(i)}\right\}=$ $\Omega_{\mathrm{rs}}^{(i)}$ and $\mathbb{E}\left\{g_{\mathrm{rp}}^{(i)}\right\}=\Omega_{\mathrm{rp}}^{(i)}$.

We assume that the secondary system utilises a two-step amplify-and-forward (AF) protocol. In the first step, the SUTx transmits the signal $\sqrt{P_{\mathrm{S}}} s_{\mathrm{S}}$ to the relays, where $P_{\mathrm{S}}$ is the SU-Tx transmit power and $s_{\mathrm{S}}$ the information symbol. During this time, the PU-Tx transmits the signal $\sqrt{P_{\mathrm{p}}} s_{\mathrm{p}}^{(1)}$, where $P_{\mathrm{p}}$ is the PU-Tx transmit power and $s_{\mathrm{p}}^{(1)}$ the information symbol. In our analysis, we assume that the SU-Tx transmit power is sufficiently attenuated by distance to be ignored at the PURx. We assume that $\mathbb{E}\left\{\left|s_{\mathrm{s}}\right|^{2}\right\}=\mathbb{E}\left\{\left|s_{\mathrm{p}}^{(1)}\right|^{2}\right\}=1$. The signal received at the $i$ th relay is given by

$$
x_{i}=\underbrace{\sqrt{P_{\mathrm{s}}} s_{\mathrm{s}} h_{\mathrm{sr}}^{*(i)}}_{\text {desired signal }}+\underbrace{\sqrt{P_{\mathrm{p}}} s_{\mathrm{p}}^{(1)} h_{\mathrm{pr}}^{*(i)}+n_{\mathrm{r}}^{(i)}}_{\text {interference }+ \text { noise }},
$$

where $n_{\mathrm{r}}^{(i)}$ is the additive white Gaussian noise (AWGN) with a variance of $\sigma_{\mathrm{r}}^{2}$ at the $i$ th relay.

In the second step, the $i$ th relay transmits the signal

$$
y_{i}=\sqrt{P_{\mathrm{s}}} s_{\mathrm{s}} h_{\mathrm{sr}}^{*(i)} w_{i}+\sqrt{P_{\mathrm{p}}} s_{\mathrm{p}}^{(1)} h_{\mathrm{pr}}^{*(i)} w_{i}+n_{\mathrm{r}}^{(i)} w_{i},
$$

where $w_{i}$ is the complex beamforming weight applied by the $i$ th relay. During this time, the PU-Tx transmits the signal $\sqrt{P_{\mathrm{p}}} s_{\mathrm{p}}^{(2)}$, where $s_{\mathrm{p}}^{(2)}$ is the information symbol and is assumed to be different to that transmitted in the first step. We assume $\mathbb{E}\left\{\left|s_{\mathrm{p}}^{(2)}\right|^{2}\right\}=1$. The signal at the SU-Rx can be expressed as

$$
\begin{aligned}
z_{s}= & \underbrace{\sqrt{P_{\mathrm{s}}} s_{\mathrm{s}}\left[\mathbf{h}_{\mathrm{sr}} \odot \mathbf{h}_{\mathrm{rs}}\right]^{H} \mathbf{w}}_{\text {desired signal }}+\underbrace{\left[\mathbf{n}_{\mathrm{r}} \odot \mathbf{h}_{\mathrm{rs}}\right]^{H} \mathbf{w}+n_{\mathrm{s}}}_{\text {noise }} \\
& +\underbrace{\sqrt{P_{\mathrm{p}}} s_{\mathrm{p}}^{(2)} h_{\mathrm{ps}}^{*}+\sqrt{P_{\mathrm{p}}} s_{\mathrm{p}}^{(1)}\left[\mathbf{h}_{\mathrm{pr}} \odot \mathbf{h}_{\mathrm{rs}}\right]^{H} \mathbf{w}}_{\text {interference }},
\end{aligned}
$$

where $\mathbf{h}_{\mathrm{sr}} \triangleq\left[h_{\mathrm{sr}}^{(1)} h_{\mathrm{sr}}^{(2)} \ldots h_{\mathrm{sr}}^{(R)}\right]^{T}, \mathbf{h}_{\mathrm{rs}} \triangleq\left[h_{\mathrm{rs}}^{(1)} h_{\mathrm{rs}}^{(2)} \ldots h_{\mathrm{rs}}^{(R)}\right]^{T}$ and $\mathbf{h}_{\mathrm{pr}} \triangleq\left[h_{\mathrm{pr}}^{(1)} h_{\mathrm{pr}}^{(2)} \ldots h_{\mathrm{pr}}^{(R)}\right]^{T}$. The signal at the PU-Rx is given by

$$
\begin{aligned}
z_{p}= & \underbrace{\sqrt{P_{\mathrm{p}}} s_{\mathrm{p}}^{(2)} h_{\mathrm{pp}}}_{\text {desired signal }}+\underbrace{\left[\mathbf{n}_{\mathrm{r}} \odot \mathbf{h}_{\mathrm{rp}}\right]^{H} \mathbf{w}+n_{\mathrm{p}}}_{\text {noise }} \\
& +\underbrace{\sqrt{P_{\mathrm{s}}} s_{\mathrm{s}}\left[\mathbf{h}_{\mathrm{sr}} \odot \mathbf{h}_{\mathrm{rp}}\right]^{H} \mathbf{w}}_{\text {SU interference }}+\underbrace{\sqrt{P_{\mathrm{p}}} s_{\mathrm{p}}^{(1)}\left[\mathbf{h}_{\mathrm{pr}} \odot \mathbf{h}_{\mathrm{rp}}\right]^{H} \mathbf{w}}_{\text {self interference }},
\end{aligned}
$$

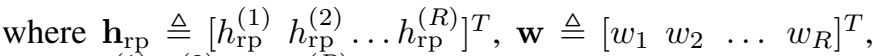
$\mathbf{n}_{\mathrm{r}} \triangleq\left[\begin{array}{llll}n_{r}^{(1)} & n_{r}^{(2)} & \ldots & n_{r}^{(R)}\end{array}\right]^{T}$ and $n_{\mathrm{s}}$ and $n_{\mathrm{p}}$ are the AWGN with powers $\sigma_{\mathrm{s}}^{2}$ and $\sigma_{\mathrm{p}}^{2}$ at the SU-Rx and PU-Rx, respectively. Note that due to the presence of the relays, the PU-Rx also receives the PU-Tx symbol from the first step, which we consider as self interference in this paper.

Using the assumption that $s_{\mathrm{s}}, s_{\mathrm{p}}^{(1)}, s_{\mathrm{p}}^{(2)}, n_{\mathrm{r}}^{(i)} \forall i, n_{\mathrm{s}}$ and $n_{\mathrm{p}}$ are all uncorrelated from each other and the availability of full CSI, and therefore considering the channel coefficients as constants, the total relay transmit power can be expressed as

$$
P_{T}=\sum_{i=1}^{R} \mathbb{E}\left\{\left|y_{i}\right|^{2}\right\}=\mathbf{w}^{H} \mathbf{E w}
$$

where $\mathbf{E}$ is defined as

$$
\mathbf{E} \triangleq P_{\mathrm{s}} \operatorname{diag}\left(\left|\mathbf{h}_{\mathrm{sr}}\right|^{2}\right)+P_{\mathrm{p}} \operatorname{diag}\left(\left|\mathbf{h}_{\mathrm{pr}}\right|^{2}\right)+\sigma_{\mathrm{r}}^{2} \mathrm{I} .
$$

Similarly, the transmit power of the $i$ th relay is given by

$$
P_{R l}^{i}=\left|\mathbf{E}_{i i}\right|^{2}\left|w_{i}\right|^{2} .
$$

The SINR at the SU-Rx is expressed as

$$
\begin{aligned}
\gamma_{\mathrm{s}} & =\frac{P_{\mathrm{s}}\left|\left[\mathbf{h}_{\mathrm{sr}} \odot \mathbf{h}_{\mathrm{rs}}\right]^{H} \mathbf{w}\right|^{2}}{P_{\mathrm{p}}\left|h_{\mathrm{ps}}\right|^{2}+P_{\mathrm{p}}\left|\left[\mathbf{h}_{\mathrm{pr}} \odot \mathbf{h}_{\mathrm{rs}}\right]^{H} \mathbf{w}\right|^{2}+\sigma_{\mathrm{r}}^{2}\left\|\mathbf{h}_{\mathrm{rs}} \odot \mathbf{w}\right\|^{2}+\sigma_{\mathrm{s}}^{2}} \\
& =\frac{\mathbf{w}^{H} \mathbf{Q w}}{P_{\mathrm{p}}\left|h_{\mathrm{ps}}\right|^{2}+\mathbf{w}^{H}(\mathbf{R}+\mathbf{V}) \mathbf{w}+\sigma_{\mathrm{s}}^{2}},
\end{aligned}
$$

where $\mathbf{Q}=P_{\mathrm{s}}\left[\mathbf{h}_{\mathrm{sr}} \odot \mathbf{h}_{\mathrm{rs}}\right]\left[\mathbf{h}_{\mathrm{sr}} \odot \mathbf{h}_{\mathrm{rs}}\right]^{H}, \mathbf{V}=\sigma_{\mathrm{r}}^{2} \operatorname{diag}\left(\left|\mathbf{h}_{\mathrm{rs}}\right|^{2}\right)$ and $\mathbf{R}=P_{\mathrm{p}}\left[\mathbf{h}_{\mathrm{pr}} \odot \mathbf{h}_{\mathrm{rs}}\right]\left[\mathbf{h}_{\mathrm{pr}} \odot \mathbf{h}_{\mathrm{rs}}\right]^{H}$. Using the following definition

$$
\begin{aligned}
I_{p} \triangleq & P_{\mathrm{s}}\left|\left[\mathbf{h}_{\mathrm{sr}} \odot \mathbf{h}_{\mathrm{rp}}\right]^{H} \mathbf{w}\right|^{2}+P_{\mathrm{p}}\left|\left[\mathbf{h}_{\mathrm{pr}} \odot \mathbf{h}_{\mathrm{rp}}\right]^{H} \mathbf{w}\right|^{2} \\
& +\sigma_{\mathrm{r}}^{2}\left\|\mathbf{h}_{\mathrm{rp}} \odot \mathbf{w}\right\|^{2},
\end{aligned}
$$

the SINR at the PU-Rx can be expressed as

$$
\gamma_{\mathrm{p}}=\frac{P_{\mathrm{p}}\left|h_{\mathrm{pp}}\right|^{2}}{I_{p}+\sigma_{\mathrm{p}}^{2}}=\frac{P_{\mathrm{p}}\left|h_{\mathrm{pp}}\right|^{2}}{\mathbf{w}^{H}(\mathbf{B}+\mathbf{C}+\mathbf{D}) \mathbf{w}+\sigma_{\mathrm{p}}^{2}},
$$

where $\mathbf{D}=\sigma_{\mathrm{r}}^{2} \operatorname{diag}\left(\left|\mathbf{h}_{\mathrm{rp}}\right|^{2}\right), \mathbf{B}=P_{\mathrm{s}}\left[\mathbf{h}_{\mathrm{sr}} \odot \mathbf{h}_{\mathrm{rp}}\right]\left[\mathbf{h}_{\mathrm{sr}} \odot \mathbf{h}_{\mathrm{rp}}\right]^{H}$ and $\mathbf{C}=P_{\mathrm{p}}\left[\mathbf{h}_{\mathrm{pr}} \odot \mathbf{h}_{\mathrm{rp}}\right]\left[\mathbf{h}_{\mathrm{pr}} \odot \mathbf{h}_{\mathrm{rp}}\right]^{H}$. 
In a cognitive radio system the secondary users are allowed to operate as long as they can guarantee a certain level of quality of service (QoS) to the primary user. Hence, in our analysis we impose an SINR constraint, $\gamma_{T}$, at the PU receiver, i.e., $\gamma_{\mathrm{p}} \geq \gamma_{\mathrm{T}}$.

\section{BEAMFORMER OPTIMISATION UNDER FULL CSI}

In this section, we aim to find the optimum beamforming weight vector, $\mathbf{w}$, such that either the total relay transmit power, $P_{T}$, is minimised or the SINR at the SU-Rx, $\gamma_{\mathrm{s}}$, is maximised while maintaining the PU receiver QoS above the threshold $\gamma_{\mathrm{T}}$. In the case where the total relay transmit power is minimised, we also impose a minimum SINR threshold, $\gamma_{\mathrm{s} \text {,min }}$, on the SU-Rx. This represents a practical limitation on the SU-Rx below which it fails to operate with acceptable performance. We also set individual maximum transmit power constraint, $P_{R l, \max }^{i}$, on each relay node when maximising the SU-Rx SINR. In practice, this constraint may be due either to regulatory or hardware limitations.

In our analysis, we assume that we are unable to control the PU's transmit power and that the PU transmits at a constant power of $P_{\mathrm{p}}$. In this section, we formulate the beamforming problem under the assumption that full CSI for all links are available which we use as the basis for the development of robust beamformers in Section IV.

\section{A. Relay Power Minimisation}

The total relay transmit power minimisation problem can be mathematically represented as

$$
\begin{array}{ll}
\min _{\mathbf{w}} & \mathbf{w}^{H} \mathbf{E w} \\
\text { s.t. } & \gamma_{\mathrm{p}} \geq \gamma_{\mathrm{T}} \\
& \gamma_{\mathrm{s}} \geq \gamma_{\mathrm{s}, \text { min }}
\end{array}
$$

Similar to other beamforming problems (see, for example [4]), it can easily be shown that constraint (9c) is satisfied with equality at the optimum, for otherwise, the optimum w could be scaled down to satisfy the constraint with equality, hence decreasing the objective function and contradicting optimality. Problem (9) is a non-convex optimisation problem, however, it can be reformulated into a convex optimisation problem. Inspired by [14], we observe that neither the objective function nor the constraints change if the beamforming vector undergoes a phase rotation. Thus, $\left[\mathbf{h}_{\mathrm{sr}} \odot \mathbf{h}_{\mathrm{rs}}\right]^{H} \mathbf{w}$ can be chosen to be real without the loss of generality. The relay power minimisation problem can therefore be restated as the following SOCP

$$
\begin{array}{ll}
\min _{\mathbf{w}} & \mathbf{w}^{H} \mathbf{E w} \\
\text { s.t. } & \sqrt{P_{\mathrm{p}}\left|h_{\mathrm{pp}}\right|^{2}} \geq \sqrt{\gamma_{\mathrm{T}}}\left\|\mathbf{v}_{1}\right\| \\
& \sqrt{P_{\mathrm{s}}}\left[\mathbf{h}_{\mathrm{sr}} \odot \mathbf{h}_{\mathrm{rs}}\right]^{H} \mathbf{w} \geq \sqrt{\gamma_{\mathrm{s}, \min }}\left\|\mathbf{v}_{2}\right\|,
\end{array}
$$

where $\mathbf{v}_{1}=\left[\sigma_{\mathrm{p}}, \sqrt{P_{\mathrm{p}}}\left[\mathbf{h}_{\mathrm{pr}} \odot \mathbf{h}_{\mathrm{rp}}\right]^{H} \mathbf{w}, \sqrt{P_{\mathrm{s}}}\left[\mathbf{h}_{\mathrm{sr}} \odot\right.\right.$ $\left.\left.\mathbf{h}_{\mathrm{rp}}\right]^{H} \mathbf{w}, \sigma_{\mathrm{r}}\left(\left[\mathbf{h}_{\mathrm{rp}} \odot \mathbf{w}\right]\right)^{T}\right]^{T}$ and $\mathbf{v}_{2}=\left[\sigma_{\mathrm{s}}, \sqrt{P_{\mathrm{p}}} h_{\mathrm{ps}}, \sigma_{\mathrm{r}}\left(\left[\mathbf{h}_{\mathrm{rs}} \odot\right.\right.\right.$ $\left.\mathbf{w}])^{T}, \sqrt{P_{\mathrm{p}}}\left[\mathbf{h}_{\mathrm{pr}} \odot \mathbf{h}_{\mathrm{rs}}\right]^{H} \mathbf{w}\right]^{T}$. In the interest of brevity, the assumed constraints $\Re\left\{\left[\mathbf{h}_{\mathrm{sr}} \odot \mathbf{h}_{\mathrm{rs}}\right]^{H} \mathbf{w}\right\} \geq 0$ and $\Im\left\{\left[\mathbf{h}_{\mathrm{sr}} \odot \mathbf{h}_{\mathrm{rs}}\right]^{H} \mathbf{w}\right\}=0$, are not explicitly stated in any of the SOCPs in this paper.

\section{B. Secondary Receiver SINR Maximisation}

The SU-Rx SINR maximisation problem is expressed as

$$
\begin{aligned}
\max _{\mathbf{w}} & \multicolumn{1}{c}{\mathbf{w}^{H} \mathbf{Q w}} \\
\text { s.t. } & \mathbf{E}_{i i}\left|w_{i}\right|^{2} \leq P_{R l, \max }^{i}, \quad i=1 \ldots R \\
& \mathbf{w}^{H} \gamma_{\mathrm{T}}(\mathbf{B}+\mathbf{C}+\mathbf{D}) \mathbf{w}+\gamma_{\mathrm{T}} \sigma_{\mathrm{p}}^{2}-P_{\mathrm{p}}\left|h_{\mathrm{pp}}\right|^{2} \leq 0(11 \mathrm{a})
\end{aligned}
$$

Using the definition $\mathbf{W} \triangleq \mathbf{w} \mathbf{w}^{H}$, problem (11) can be restated in the epigraph form [13] as

$$
\begin{array}{rl}
\max _{\mathbf{W}, t} & t \\
\text { s.t. } & \operatorname{tr}((\mathbf{Q}-t(\mathbf{R}+\mathbf{V})) \mathbf{W}) \geq\left(P_{\mathrm{p}}\left|h_{\mathrm{ps}}\right|^{2}+\sigma_{\mathrm{s}}^{2}\right) t \\
& \operatorname{rank}(\mathbf{W})=1 \\
& \mathbf{W} \succeq 0,(11 \mathrm{~b}) \text { and (11c) }
\end{array}
$$

Due to the non-convex constraints (12b) and (12c), problem (12) is a non-convex optimisation problem. We apply the idea of semidefinite relaxation (SDR) $[13,15]$ and relax problem (12) by removing the non-convex rank-one constraint (12c). The resulting relaxed problem is still non-convex as constraint (12b) remains to be dealt with. In $[4,13]$, it was shown that in problems of this nature, for any fixed value of $t$ the set of feasible $\mathbf{W}$ is convex and hence the relaxed problem is quasi convex. Therefore, for some given $t$, problem (12) can be expressed as the following convex feasibility problem

$$
\text { find } \mathbf{W} \quad \text { s.t. } \mathbf{W} \succeq 0,(11 b),(11 \mathrm{c}) \text { and }(12 b) \text {. }
$$

The bisection method [13] is a commonly used technique for solving convex feasibility problems like problem (13). Upon completion of the bisection algorithm, one needs to recover the optimum beamforming vector, $\mathbf{w}^{*}$, from $\mathbf{W}$. If $\mathbf{W}$ is rankone, then $\mathbf{w}^{*}$ can be chosen to be the principle eigenvector of $\mathbf{W}$. For the case where $\mathbf{W}$ has rank higher than one, the well known Gaussian randomisation technique [15] can be used to recover a good rank-one approximation, however, in our extensive numerical simulations we have never obtained a solution that had a rank higher than one. This behaviour is in agreement with those published in a number of other works on beamforming (see, for example [4]).

\section{ROBUST BEAMFORMER OPTIMISATION UNDER PARTIAL CSI}

In practice, full CSI for all links are seldom available and the assumption of full CSI may be overly idealistic. We consider a CR relay network with a very loose cooperation with the primary network. We assume that only mean channel powers of the PU transmitter to PU receiver and the relays to PU receiver links are available, i.e., only $\Omega_{\mathrm{pp}}$ and $\Omega_{\mathrm{rp}}^{(i)} \forall i$ for the aforementioned links are available. Furthermore, we assume that the channel for the SU-Tx to SU-RI and SU-RI to SU-Rx links are accurately known through the SU's channel estimation procedure and those between the PU-Tx and SU-RI can be accurately measured, for example through knowledge of the PU pilot symbols.

In our formulation we consider the PU outage probability as a QoS parameter. In the system under consideration, outage 
occurs when the PU SINR, $\gamma_{\mathrm{p}}$, falls below the PU SINR threshold, $\gamma_{\mathrm{T}}$. The outage probability is expressed as

$$
\begin{aligned}
\mathrm{P}_{\mathrm{o}} & =\operatorname{Pr}\left\{\gamma_{\mathrm{p}} \leq \gamma_{\mathrm{T}}\right\} \\
& =\operatorname{Pr}\left\{P_{\mathrm{p}}\left|h_{\mathrm{pp}}\right|^{2}-\gamma_{\mathrm{T}} \mathbf{w}^{H}(\mathbf{B}+\mathbf{C}+\mathbf{D}) \mathbf{w} \leq \gamma_{\mathrm{T}} \sigma_{\mathrm{p}}^{2}\right\} .
\end{aligned}
$$

Hence, given a maximum allowable outage probability, $\mathrm{P}_{\mathrm{o} \text {, max }}$, constraints $(10 \mathrm{~b})$ and $(11 \mathrm{c})$ are replaced with $\mathrm{P}_{\mathrm{o}} \leq$ $\mathrm{P}_{\mathrm{o} \text {, max }}$.

We observe that in (14), we are dealing with a probability density function (PDF) that is given by the difference of two random variables, namely, $P_{\mathrm{p}}\left|h_{\mathrm{pp}}\right|^{2}$ and $\gamma_{\mathrm{T}} \mathbf{w}^{H}(\mathbf{B}+\mathbf{C}+\mathbf{D}) \mathbf{w}$. It is easily shown that $P_{\mathrm{p}}\left|h_{\mathrm{pp}}\right|^{2}$ has an exponential distribution with a mean of $P_{\mathrm{p}} \Omega_{\mathrm{pp}}$. The probability distribution of $\gamma_{\mathrm{T}} \mathbf{w}^{H}(\mathbf{B}+\mathbf{C}+\mathbf{D}) \mathbf{w}$ can be found using the following lemma.

Lemma 1. If $\mathrm{x} \in \mathcal{C}^{R \times 1}$ is distributed as $\mathrm{x} \sim \mathcal{N}_{\mathcal{C}}(\mathbf{0}, \boldsymbol{\Sigma})$, then for any deterministic positive semidefinite Hermitian matrix $\mathbf{A} \in \mathcal{C}^{R \times R}$, the PDF of the random variable $\psi=\mathbf{x}^{H} \mathbf{A} \mathbf{x}$, $\psi \geq 0$, is given by

$$
f(\psi)=\left[\prod_{i=1}^{N} \lambda_{i}\right] \sum_{j=1}^{N} \frac{\exp \left(-\lambda_{j} \psi\right)}{\prod_{k=1, k \neq j}^{N}\left(\lambda_{k}-\lambda_{j}\right)},
$$

where $\lambda_{i}=1 / \Lambda_{i}$, and $\Lambda_{i}, i=1 \ldots N \leq R$ are the non-zero eigenvalues of $\boldsymbol{\Sigma} \mathbf{A}$. Note that this is precisely the distribution of the sum of $N$ exponentially distributed independent random variables, each with a mean of $\Lambda_{i}$.

Proof: Note that $\mathbf{x}^{H} \mathbf{A x}$ is commonly known as a quadratic form in normal random variables [16]. We first define $\mathbf{y}=\boldsymbol{\Sigma}^{-\frac{1}{2}} \mathbf{x}$. It is easily verified that $\mathbf{y} \sim \mathcal{N}_{\mathcal{C}}(\mathbf{0}, \mathbf{I})$. Using an orthogonal $R \times R$ matrix $\mathbf{P}$ that diagonalises $\boldsymbol{\Sigma}^{\frac{1}{2}} \mathbf{A} \boldsymbol{\Sigma}^{\frac{1}{2}}$ or equivalently $\boldsymbol{\Sigma} \mathbf{A}$, i.e., $\mathbf{P}^{H} \boldsymbol{\Sigma} \mathbf{A P}=\operatorname{diag}\left(\Lambda_{1}, \Lambda_{2}, \ldots, \Lambda_{R}\right)$ and $\Lambda_{1}, \Lambda_{2}, \ldots, \Lambda_{R}$ are the eigenvalues of $\boldsymbol{\Sigma} \mathbf{A}, \psi$ can then be expressed as

$$
\begin{aligned}
\psi & =\mathbf{y}^{H} \boldsymbol{\Sigma}^{\frac{1}{2}} \mathbf{A} \boldsymbol{\Sigma}^{\frac{1}{2}} \mathbf{y}=\left(\mathbf{P}^{H} \mathbf{y}\right)^{H} \mathbf{P}^{H} \boldsymbol{\Sigma}^{\frac{1}{2}} \mathbf{A} \boldsymbol{\Sigma}^{\frac{1}{2}} \mathbf{P}\left(\mathbf{P}^{H} \mathbf{y}\right) \\
& =\operatorname{tr}\left(\operatorname{diag}\left(\Lambda_{1}, \Lambda_{2}, \ldots, \Lambda_{R}\right) \mathbf{P}^{H} \mathbf{y} \mathbf{y}^{H} \mathbf{P}\right) \\
& =\sum_{i=1}^{N} \Lambda_{i}\left|\left(\mathbf{P}^{H} \mathbf{y}\right)_{i}\right|^{2},
\end{aligned}
$$

where $N \leq R$ is the number of non-zero eigenvalues of $\boldsymbol{\Sigma} \mathbf{A}$ and $\left(\mathbf{P}^{H} \mathbf{y}\right)_{i}$ is the $i$ th element of the vector $\mathbf{P}^{H} \mathbf{y}$. Since $\mathbf{P}$ is an orthogonal matrix, it is easily shown that $\mathbf{P}^{H} \mathbf{y} \sim \mathcal{N}_{\mathcal{C}}(\mathbf{0}, \mathbf{I})$, and therefore, $\left|\left(\mathbf{P}^{H} \mathbf{y}\right)_{i}\right|^{2}$ is an exponentially distributed random variable with a mean of $\Lambda_{i}$. Hence, (16) is a sum of $N$ exponentially distributed independent random variables, each with a mean of $\Lambda_{i}$, whose PDF is given by (15). The derivation of the PDF of the sum of $N$ exponentially distributed independent random variables has appeared in many texts, we refer the interested reader to [17] and references therein.

From the definition of $\mathbf{B}$, we note that it can be expressed as $\mathbf{B}=\mathbf{b b}^{H}$, where $\mathbf{b}=\sqrt{P_{\mathrm{s}}}\left[\mathbf{h}_{\mathrm{sr}} \odot \mathbf{h}_{\mathrm{rp}}\right]$. Furthermore, by defining $\mathbf{W}=\mathbf{w} \mathbf{w}^{H}$ we see that $\mathbf{w}^{H} \mathbf{B} \mathbf{w}=\mathbf{b}^{H} \mathbf{W} \mathbf{b}$. Using Lemma 1 and exploiting the fact that $\mathbf{W}$ is a rank-one matrix, we have that $\gamma_{\mathrm{T}} \mathbf{w}^{H} \mathbf{B w}$ is exponentially distributed with a mean of $\operatorname{tr}\left(\boldsymbol{\Sigma}_{B} \mathbf{W}\right)$, where $\boldsymbol{\Sigma}_{B}$ is the covariance matrix of $\sqrt{\gamma_{\mathrm{T}}} \mathbf{b}$ and is expressed as

$$
\boldsymbol{\Sigma}_{B}=\gamma_{\mathrm{T}} P_{\mathrm{s}} \operatorname{diag}\left(\boldsymbol{\Omega}_{\mathrm{rp}} \odot\left|\mathbf{h}_{\mathrm{sr}}\right|^{2}\right) .
$$

where $\boldsymbol{\Omega}_{\mathrm{rp}}=\left[\begin{array}{lll}\Omega_{\mathrm{rp}}^{1} & \Omega_{\mathrm{rp}}^{2} & \ldots \Omega_{\mathrm{rp}}^{R}\end{array}\right]^{T}$. Similarly, $\gamma_{\mathrm{T}} \mathbf{w}^{H} \mathbf{C w}$ also has an exponential distribution with a mean of $\operatorname{tr}\left(\boldsymbol{\Sigma}_{C} \mathbf{W}\right)$, where $\Sigma_{C}$ is the covariance matrix defined as

$$
\boldsymbol{\Sigma}_{C}=\gamma_{\mathrm{T}} P_{\mathrm{p}} \operatorname{diag}\left(\boldsymbol{\Omega}_{\mathrm{rp}} \odot\left|\mathbf{h}_{\mathrm{pr}}\right|^{2}\right) \text {. }
$$

Since $\mathbf{D}$ is a diagonal matrix, we have $\gamma_{\mathrm{T}} \mathbf{w}^{H} \mathbf{D w}=$ $\gamma_{\mathrm{T}} \sigma_{\mathrm{r}}^{2} \sum_{i=1}^{R} \mathbf{W}_{i i}\left|h_{\mathrm{rp}}^{(i)}\right|^{2}$, which is recognised as a sum of $R$ exponentially distributed random variables and as such, the PDF is given by (15) whereby $N=R$ and $\lambda_{i}=1 /\left(\gamma_{\mathrm{T}} \sigma_{\mathrm{r}}^{2} \Omega_{\mathrm{rp}}^{(i)} \mathbf{W}_{i i}\right)$, $i=1 \ldots R$.

Finally, from the above analysis, we see that $\gamma_{\mathrm{T}} \mathbf{w}^{H}(\mathbf{B}+\mathbf{C}+$ D)w is the sum of $R+2$ exponentially distributed independent random variables and the PDF is once again given by (15), with $N=R+2, \lambda_{i}=1 /\left(\gamma_{\mathrm{T}} \sigma_{\mathrm{r}}^{2} \Omega_{\mathrm{rp}}^{(i)} \mathbf{W}_{i i}\right), i=1 \ldots R, \lambda_{R+1}=$ $1 / \operatorname{tr}\left(\boldsymbol{\Sigma}_{B} \mathbf{W}\right)$ and $\lambda_{R+2}=1 / \operatorname{tr}\left(\boldsymbol{\Sigma}_{C} \mathbf{W}\right)$.

We note that the PDF in (14) is that of a difference between an exponential random variable and the sum of $R+2$ exponentially distributed random variables, and therefore the outage probability is commonly known to have the following form (see, for example, [17])

$$
\mathrm{P}_{\mathrm{o}}=1-\exp \left(-\frac{\gamma_{\mathrm{T}} \sigma_{\mathrm{p}}^{2}}{P_{\mathrm{p}} \Omega_{\mathrm{pp}}}\right) \prod_{i=1}^{R+2}\left(\frac{1}{1+\frac{1}{P_{\mathrm{p}} \Omega_{\mathrm{pp}} \lambda_{i}}}\right),
$$

where $\lambda_{i}$ is as defined previously. Using (19), the outage probability constraint can be expressed as

$$
\prod_{i=1}^{R+2}\left(1+\frac{1}{P_{\mathrm{p}} \Omega_{\mathrm{pp}} \lambda_{i}}\right) \leq \frac{\exp \left(-\frac{\gamma_{\mathrm{T}} \sigma_{\mathrm{p}}^{2}}{P_{\mathrm{p}} \Omega_{\mathrm{pp}}}\right)}{1-\mathrm{P}_{\mathrm{o}, \max }}
$$

An important observation in the above constraint is that it is dependent only on the diagonal elements of $\mathbf{W}$, i.e., dependent only on the beamformer transmit power. This is a fairly intuitive result since phase information of SU-Rl to PU-Rx link is not available and, therefore, power control is the only degree of freedom available to the beamformer to control the amount of interference to the PU-Rx. Note that constraint (20) is non-convex, and is difficult to handle. For this reason, we propose to use the geometric-arithmetic mean inequality and replace the left hand side of (20) with its upper bound. The geometric-arithmetic mean inequality is expressed as

$$
\prod_{i=1}^{R+2}\left(1+\frac{1}{P_{\mathrm{p}} \Omega_{\mathrm{pp}} \lambda_{i}}\right)^{\frac{1}{R+2}} \leq \frac{1}{R+2} \sum_{i=1}^{R+2}\left(1+\frac{1}{P_{\mathrm{p}} \Omega_{\mathrm{pp}} \lambda_{i}}\right) .
$$

Using the above inequality, the convex outage probability constraint is thus

$$
\sum_{i=1}^{R+2}\left(1+\frac{1}{P_{\mathrm{p}} \Omega_{\mathrm{pp}} \lambda_{i}}\right) \leq(R+2)\left(\frac{\exp \left(-\frac{\gamma_{\mathrm{T}} \sigma_{\mathrm{p}}^{2}}{P_{\mathrm{p}} \Omega_{\mathrm{pp}}}\right)}{1-\mathrm{P}_{\mathrm{o}, \max }}\right)^{\frac{1}{R+2}}
$$

By using the upper bound, the constraint is being tightened and the ramifications of this on the optimum solution are discussed later in this section. Meanwhile, we present the robust optimisation problems by directly using (21). 
Through straightforward manipulation, (21) can be rewritten as

$$
\begin{aligned}
& \frac{1}{P_{\mathrm{p}} \Omega_{\mathrm{pp}}} \mathbf{w}^{H}\left(\boldsymbol{\Sigma}_{B}+\boldsymbol{\Sigma}_{C}+\gamma_{\mathrm{T}} \sigma_{\mathrm{r}}^{2} \operatorname{diag}\left(\boldsymbol{\Omega}_{\mathrm{rp}}\right)\right) \mathbf{w} \\
& +(R+2)\left(1-\left(\frac{\exp \left(-\frac{\gamma_{\mathrm{T}} \sigma_{\mathrm{p}}^{2}}{P_{\mathrm{p}} \Omega_{\mathrm{pp}}}\right)}{1-\mathrm{P}_{\mathrm{o}, \max }}\right)^{\frac{1}{R+2}}\right) \leq 0,
\end{aligned}
$$

The equivalent SOCP constraint is given by

$$
\begin{aligned}
& \sqrt{(R+2)\left(\left(\exp \left(-\frac{\gamma_{\mathrm{T}} \sigma_{\mathrm{p}}^{2}}{P_{\mathrm{p}} \Omega_{\mathrm{pp}}}\right) /\left(1-\mathrm{P}_{\mathrm{o}, \max }\right)\right)^{\frac{1}{R+2}}-1\right)} \\
& \geq \sqrt{\gamma_{\mathrm{T}} /\left(P_{\mathrm{p}} \Omega_{\mathrm{pp}}\right)}\left\|\mathbf{v}_{3}\right\|
\end{aligned}
$$

where $\quad \mathbf{v}_{3}=\left[\sigma_{\mathrm{r}}\left[\boldsymbol{\Omega}_{\mathrm{rp}}^{1 / 2} \odot \mathbf{w}\right]^{T}, \sqrt{P_{\mathrm{s}}}\left[\boldsymbol{\Omega}_{\mathrm{rp}}^{1 / 2} \odot \mathbf{h}_{\mathrm{sr}} \odot\right.\right.$ $\left.\mathbf{w}]^{T}, \sqrt{P_{\mathrm{s}}}\left[\boldsymbol{\Omega}_{\mathrm{rp}}^{1 / 2} \odot \mathbf{h}_{\mathrm{sr}} \odot \mathbf{w}\right]^{T}\right]^{T}$ and $\boldsymbol{\Omega}_{\mathrm{rp}}^{1 / 2}$ is the element-wise square root of the vector $\Omega_{\mathrm{rp}}$.

The robust SU-Rl power minimisation SOCP can therefore be expressed as

$$
\min _{\mathbf{w}} \mathbf{w}^{H} \mathbf{E w}, \quad \text { s.t. } \quad \text { (23) and (10c). }
$$

Using constraint (21), the robust SU-Rx SINR maximisation problem can again be expressed as the convex feasibility problem

$$
\text { find } \mathbf{W}, \quad \text { s.t. } \mathbf{W} \succeq 0,(11 b),(12 b) \text { and (21), }
$$

which can be solved using the methods described in Section III-B.

As previously mentioned, using the outage probability upper bound results in tightening of the constraint. In the SU-RI power minimisation problem, this tightening may result in some feasible problems becoming infeasible. Likewise, the SU-Rx SINR maximisation problem may become infeasible or the solution obtained may be sub-optimal.

Recalling that at the optimum, constraint (10c) is satisfied with equality, therefore, if the robust SU-Rl power minimisation problem (24) is feasible then the solution obtained is the optimum. On the other hand, if the problem is infeasible due to the tightened outage probability constraint then we need to determine if a feasible solution can be obtained by relaxing the constraint. This corresponds to finding the minimum relaxed outage probability specification, $\tilde{\mathrm{P}}_{\mathrm{o}, \max } \geq \mathrm{P}_{\mathrm{o}, \max }$, that satisfies (20). We have developed an efficient iterative method utilising the bisection technique for finding $\tilde{\mathrm{P}}_{\mathrm{o}, \max }$. In each iteration, problem (24) is solved with the relaxed outage probability specification and the solution is used in (19) to calculate the exact outage probability, $\mathrm{P}_{\mathrm{o}}$, attained. $\mathrm{P}_{\mathrm{o}}$ is then compared with $\mathrm{P}_{\mathrm{o}, \max }$ to determine if (20) is satisfied. The iterations continue until the minimum $\tilde{\mathrm{P}}_{\mathrm{O}, \max }$ is found.

An important observation in the robust SU-Rx SINR maximisation problem (25) is that, at the optimum, either the outage probability constraint or all of the transmit power constraints will be satisfied with equality. This is because both the SU-Rx SINR and the outage probability are increasing functions of relay transmit power and, therefore, the transmit power will be increased until either the outage probability constraint is met with equality or the maximum transmit power budget is met. Using this observation, we have developed an iterative algorithm utilising the bisection technique that can be used to find the optimum beamformer. The algorithm finds the minimum relaxed outage probability specification, $\tilde{\mathrm{P}}_{\mathrm{o}, \max } \geq \mathrm{P}_{\mathrm{o}, \max }$, that either satisfies (20) with equality or satisfies (11b) $\forall i$ with equality while satisfying (20).

Although the iterative algorithms proposed provide the optimum beamforming weights, through our extensive numerical simulations we have found that the solutions obtained by directly solving problems (24) and (25) with the tightened outage probability constraint are very close to the optimum and in practice it is not necessary to use the iterative algorithms.

\section{Simulation Results and Discussion}

We illustrate the performance of our proposed methods through numerical simulations in i.i.d. Rayleigh flat-fading channels. We consider a system with $R=8$ relay nodes. In all simulations we have set $P_{\mathrm{p}}=P_{\mathrm{s}}=30 \mathrm{dBm}, P_{R l, \text { max }}^{i}=30$ $\mathrm{dBm} \forall i, \gamma_{\mathrm{T}}=5 \mathrm{~dB}$ and the noise power at each receiver is assumed to be $-30 \mathrm{dBm}$. The maximum PU outage probability, $\mathrm{P}_{\mathrm{o}, \max }$, is set to $5 \%$. Channel powers of the direct paths, i.e., $\Omega_{\mathrm{pp}}, \Omega_{\mathrm{sr}}$ and $\Omega_{\mathrm{rs}}$, are set to $5 \mathrm{~dB}$. For our simulations we set the signal channel to interference channel ratio to 10 $\mathrm{dB}$, i.e., $\Omega_{\mathrm{pp}} / \Omega_{\mathrm{rp}}^{(i)}=\Omega_{\mathrm{sr}}^{(i)} / \Omega_{\mathrm{pr}}^{(i)}=\Omega_{\mathrm{rs}}^{(i)} / \Omega_{\mathrm{ps}}=10 \mathrm{~dB} \forall i$. Simulations for the total relay power minimisation problem have $\gamma_{\mathrm{s}, \min }=5 \mathrm{~dB}$. Results are compared against the full CSI and worst case designs.

The worst case beamformer is designed such that the SINR at the PU-Rx is above the threshold $\gamma_{\mathrm{T}}$, for every possible realisation of $h_{\mathrm{pp}}$ and $\mathbf{h}_{\mathrm{rp}}$. Since instantaneous realisations of $h_{\mathrm{pp}}$ and $\mathbf{h}_{\mathrm{rp}}$ are not available, our worst case designs solve problems (9) and (11) based on the expected value of (8). Note that (8) is at its minimum when $\left|h_{\mathrm{pp}}\right|^{2}=\Omega_{\mathrm{pp}}-\epsilon_{1}$ and $\left|h_{\mathrm{rp}}^{(i)}\right|^{2}=$ $\Omega_{\mathrm{rp}}+\epsilon_{2} \forall i$, for some appropriately chosen values of $\epsilon_{1}, \epsilon_{2} \geq 0$. The worst case beamformer ensures that this minimum value is always above the threshold $\gamma_{\mathrm{T}}$. To provide a fair comparison with methods proposed in this paper, $\epsilon_{1}$ and $\epsilon_{2}$ are chosen such that $\operatorname{Pr}\left\{\left|h_{\mathrm{pp}}\right|^{2} \geq \Omega_{\mathrm{pp}}-\epsilon_{1}\right\} \prod_{i=1}^{R} \operatorname{Pr}\left\{\left|h_{\mathrm{rp}}^{(i)}\right|^{2} \leq \Omega_{\mathrm{rp}}+\epsilon_{2}\right\}=$ $1-\mathrm{P}_{\mathrm{o}, \max }$.

In Fig. 2, results are provided for the CDF of the PU-Rx SINR. We see that the required probability, that the resulting PU-Rx SINR is below $5 \mathrm{~dB}$, is satisfied by both robust optimisation schemes proposed in this paper. Results also show that the iterative algorithms proposed have the same performance as the non-iterative algorithms. The probability that the SINR is below $5 \mathrm{~dB}$ in the worst case approach is almost zero.

The resulting SU-Rx SINR by solving problem (25) is shown in Fig. 3 along with the full CSI and the worst case solutions. The performance loss due to partial CSI is clearly visible. The worst case design provides the weakest received signal power because it is optimised to protect the PU-Rx on every possible CSI realisation in the chosen set. 


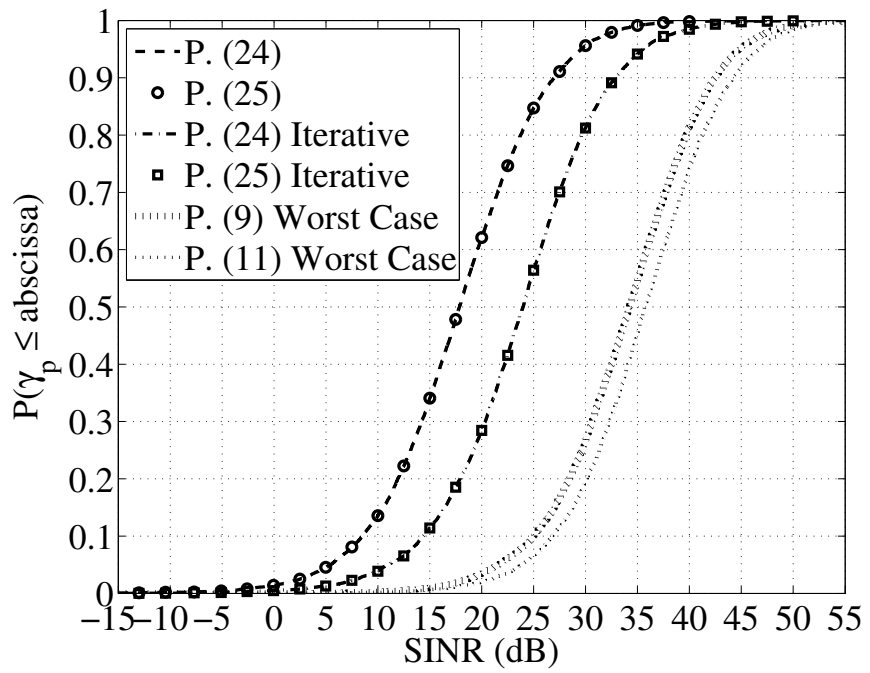

Fig. 2. SINR at the PU

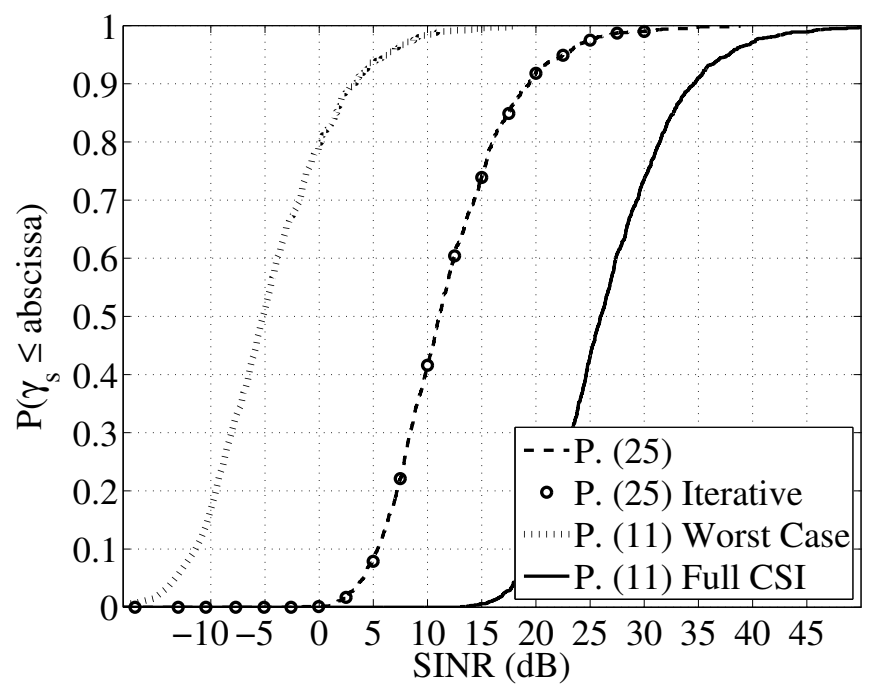

Fig. 3. SINR at the SU

Fig. 4 illustrates the CDF of the total relay transmit power obtained by solving problem (24) along with the full CSI and the worst case solutions. Being very aggressive in protecting the PU-Rx, the worst case approach transmits the least amount of power.

\section{CONCLUSIONS}

In this paper, we have studied a statistically robust cooperative beamformer for a CR relay network under the assumption of partial CSI. We have shown that the total relay power minimisation problem can be solved using SOCP and that the cognitive destination SINR maximisation problem can be stated as a convex feasibility problem using probabilistic constraints. We have proposed iterative algorithms for obtaining optimum results however, in practice, it is not necessary to use these iterative algorithms as the performance improvements are not significant.

\section{REFERENCES}

[1] P. A. Dmochowski, H. A. Suraweera, P. J. Smith, and M. Shafi, "Impact of channel knowledge on cognitive radio system capacity," in Proc. IEEE VTC2010-Fall, September 2010, pp. 1-5.

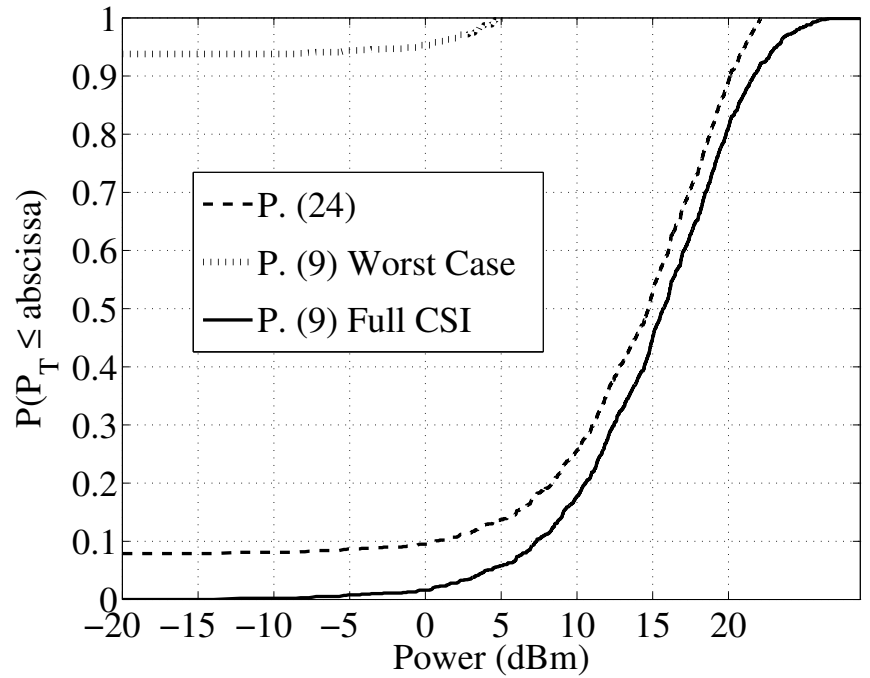

Fig. 4. Total Relay Transmit Power CDF

[2] M. Shafi, H. A. Suraweera, P. J. Smith, and M. Faulkner, "Capacity limits and performance analysis of cognitive radio with imperfect channel knowledge," IEEE Trans. on Veh. Technol., January 2010.

[3] A. Sendonaris, E. Erkip, and B. Aazhang, "User cooperation diversity. part i. system description," IEEE Trans. Commun., vol. 51, no. 11, pp. 1927 - 1938, nov. 2003.

[4] V. Havary-Nassab, S. Shahbazpanahi, A. Grami, and Z. Luo, "Distributed beamforming for relay networks based on second-order statistics of the channel state information," IEEE Trans. Signal Proc., vol. 56, no. 9 , pp. 4306-4316, Sept. 2008.

[5] G. Zheng, K.-K. Wong, A. Paulraj, and B. Ottersten, "Robust collaborative-relay beamforming," IEEE Trans. Sig. Process., vol. 57, no. 8, pp. $3130-3143$, aug. 2009.

[6] R. Zhang, C. C. Chai, and Y.-C. Liang, "Joint beamforming and power control for multiantenna relay broadcast channel with qos constraints," IEEE Trans. Sig. Process., vol. 57, no. 2, pp. 726 -737, feb. 2009.

[7] K. Hamdi, K. Zarifi, K. B. Letaief, and A. Ghrayeb, "Beamforming in relay-assisted cognitive radio systems: A convex optimization approach," in Proc. IEEE ICC 2011, june 2011, pp. 1 -5.

[8] V. Asghari and S. Aissa, "Performance of cooperative spectrum-sharing systems with amplify-and-forward relaying," IEEE Trans. Wireless Commun., vol. 11, no. 4, pp. 1295 -1300, april 2012.

[9] J. Liu, W. Chen, Z. Cao, and Y. Zhang, "Cooperative beamforming for cognitive radio networks: A cross-layer design," IEEE Trans. Commun., vol. 60 , no. 5, pp. $1420-1431$, may 2012.

[10] C.-K. Wen, J.-C. Chen, and P. Ting, "Robust transmitter design for amplify-and-forward mimo relay systems exploiting only channel statistics," Wireless Communications, IEEE Transactions on, vol. 11, no. 2, pp. $668-682$, february 2012.

[11] P. Ubaidulla and S. Aissa, "Robust distributed cognitive relay beamforming," in Proc. VTC Spring 2012, may 2012, pp. $1-5$.

[12] B. K. Chalise, S. Shahbazpanahi, A. Czylwik, and A. B. Gershman, "Robust downlink beamforming based on outage probability specifications," IEEE Trans. Wireless Commun., vol. 6, no. 10, pp. 3498-3503, Oct. 2007.

[13] S. Boyd and L. Vandenberghe, Convex Optimization. Cambridge University Press, 2009.

[14] M. Bengtsson and B. Ottersten, Handbook on Antennas in Wireless Communications. CRC, 2002, ch. Optimal and suboptimal transmit beamforming.

[15] Z. Luo and T. Chang, Convex Optimization in Signal Processing and Communications. Cambridge University Press, 2010, ch. 4 SDP relaxation of homogeneous quadratic optimization: approximation bounds and applications, pp. 117-165.

[16] A. M. Mathai and S. B. Provost, Quadratic Forms In Random Variables, Theory and Applications. Marcel Dekker, INC., 1992.

[17] S. Kandukuri and S. Boyd, "Optimal power control in interference limited fading wireless channels with outage probability specifications," IEEE Trans. Wireless Commun., vol. 1, no. 1, pp. 46-55, January 2002. 